\title{
An efficient synthesis of pyrazolo[3,4-b]pyridine derivatives under microwave irradiation
}

\author{
Xiang Zou, ${ }^{\mathrm{a}, \mathrm{b}}$ Shujiang Tu, ${ }^{\mathrm{a}}$ Feng Shi, ${ }^{\mathrm{a}}$ and Jianing $\mathrm{Xu}{ }^{\mathrm{a}}$ \\ ${ }^{a}$ Department of Chemistry, Xuzhou Normal University, Key Laboratory of Biotechnology on \\ Medical Plant, Xuzhou, Jiangsu 221009, PR China \\ ${ }^{b}$ Department of Chemistry, Lianyungang Normal University, Lianyungang, \\ Jiangsu 222006, PR China \\ E-mail: laotu2001@263.net
}

(received 15 Sep 05; accepted 9 Jan 06; published on the web 13 Jan 06)

\begin{abstract}
A series of pyrazolo[3,4-b]pyridine derivatives were synthesized by the reaction of aminopyrazole with chalcones in the presence of $\mathrm{ZnCl}_{2}$ under microwave irradiation. The reaction was completed in $8-12$ min with $85-95 \%$ yields.
\end{abstract}

Keywords: Microwave irradiation, pyrazolo[3,4-b]pyridine

\section{Introduction}

The pyrazolo $[3,4-b]$ pyridine system has shown many interesting biological and pharmacological properties, such as antitubercular activity, ${ }^{1,2}$ activity against gram positive and negative bacteria, ${ }^{3}$ and ACTH (Adrenocorticotropic hormone)-releasing factor (CRF (Corticotropin-releasing facter)) antagonist activity. CRF antagonists proved to be effective in the treatment of a wide variety of stress-related illnesses, such as depression, gastrointestinal diseases, anorexia nervosa, haemorrhaged stress, drug and alcohol withdrawal symptoms. ${ }^{4}$

Due to the importance of pyrazolo[3,4-b]pyridines, much work has been done over the years. The most important synthetic method is the condensation of aminopyrazole with $\alpha, \beta$ unsaturated compounds reported by J. Quiroga. ${ }^{5-14}$ The reaction proceeds generally in two steps, in which dihydropyrazolopyridines I are first obtained by the condensation of aminopyrazole with chalcones using traditional heating in $47-70 \%$ yields, and are then further dehydrogenated by NBS to give the desired compounds in $60-80 \%$ yields (Scheme 1 ). ${ }^{5,8}$ 

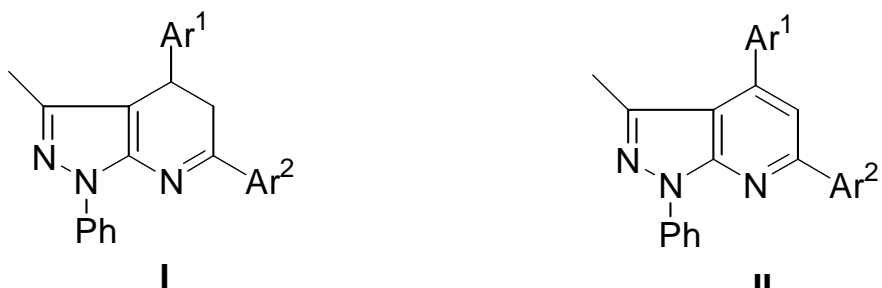

II

\section{Scheme 1}

However, we found that some chalcones such as $\mathbf{2 b}, \mathbf{2} \mathbf{g}$ and $\mathbf{2} \mathbf{i}$ did not react or react very sluggishly under these conditions. Besides, this two-step reaction has the drawback of long reaction times and a quite low total yield. In recent years, microwave techniques have developed rapidly in organic synthesis due to shorter reaction times, higher yields, easy work-up and environmentally friendliness. ${ }^{15}$ Therefore, we investigated the reaction under microwave irradiation and found that the catalyst $\mathrm{ZnCl}_{2}$ played a very important role in the above condensation reaction. At the same time, aromatized products 3 could be obtained in one step.

In this paper, we would like to report this efficient synthetic method of pyrazolo[3,4$b]$ pyridines 3 by the reaction of aminopyrazole with chalcones in one step under microwave irradiation in the presence of $\mathrm{ZnCl}_{2}$ leading to higher yields and shorter reaction times (Scheme 2).

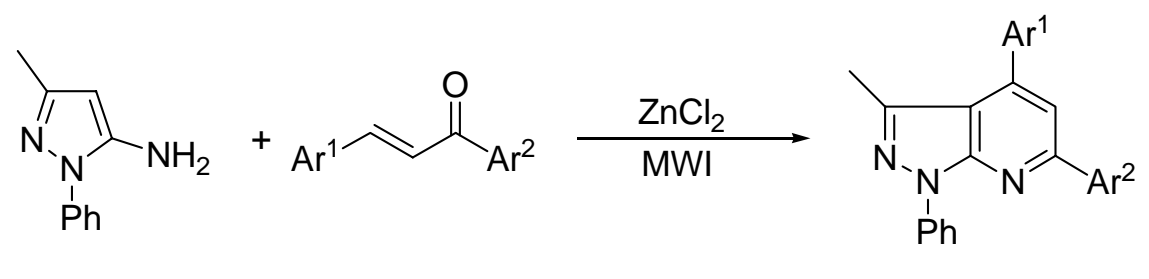

1

2

3

Scheme 2 The synthesis of pyrazolo[3,4-b]pyridine using $\mathrm{ZnCl}_{2}$ as catalyst.

\section{Results and Discussion}

The results are shown in Table 1. The reaction was completed within 8-12 min with high yields ranging from $85-95 \%$. For comparison, this reaction was carried out at $100{ }^{\circ} \mathrm{C}$ under traditional heating conditions for $3 \mathrm{~h}$, leading to lower yields $(70 \%)$. It is obvious that the procedure under microwave irradiation has the advantages of a short routine, a good yield and a convenient workup. The procedure is also more environmentally friendly compared to traditional heating. 
Table 1. Syntheses of compounds 3 under microwave irradiation

\begin{tabular}{cllccc}
\hline Entry & \multicolumn{1}{c}{$\mathrm{Ar}^{1}$} & \multicolumn{1}{c}{$\mathrm{Ar}^{2}$} & Time (min) & $\mathrm{Mp}\left({ }^{\circ} \mathrm{C}\right)$ & Yield (\%) \\
\hline 3a & $4-\mathrm{BrC}_{6} \mathrm{H}_{4}$ & $2-\mathrm{ClC}_{6} \mathrm{H}_{4}$ & 8 & $182-184$ & 90 \\
3b & $4-\mathrm{BrC}_{6} \mathrm{H}_{4}$ & $4-\mathrm{CH}_{3} \mathrm{C}_{6} \mathrm{H}_{4}$ & 10 & $181-182$ & 92 \\
3c & $4-\mathrm{FC}_{6} \mathrm{H}_{4}$ & $4-\mathrm{CH}_{3} \mathrm{C}_{6} \mathrm{H}_{4}$ & 8 & $155-156$ & 95 \\
3d & $3,4-\mathrm{Cl}_{2} \mathrm{C}_{6} \mathrm{H}_{3}$ & $4-\mathrm{CH}_{3} \mathrm{OC}_{6} \mathrm{H}_{4}$ & 10 & $169-170$ & 85 \\
3e & $3-\mathrm{NO}_{2} \mathrm{C}_{6} \mathrm{H}_{4}$ & $4-\mathrm{CH}_{3} \mathrm{OC}_{6} \mathrm{H}_{4}$ & 12 & $219-221$ & 85 \\
$\mathbf{3 f}$ & $4-\mathrm{ClC}_{6} \mathrm{H}_{4}$ & $2-\mathrm{OCH}_{3} \mathrm{C}_{6} \mathrm{H}_{4}$ & 12 & $158-160$ & 86 \\
3g & $4-\mathrm{CH}_{3} \mathrm{OC}_{6} \mathrm{H}_{4}$ & $4-\mathrm{CH}_{3} \mathrm{C}_{6} \mathrm{H}_{4}$ & 12 & $168-169$ & 89 \\
3h & $4-\mathrm{ClC}_{6} \mathrm{H}_{4}$ & $4-\mathrm{FC}_{6} \mathrm{H}_{4}$ & 8 & $158-159$ & 90 \\
$3 \mathbf{3 i}$ & $4-\mathrm{CH}_{3} \mathrm{OC}_{6} \mathrm{H}_{4}$ & $2-\mathrm{ClC}_{6} \mathrm{H}_{4}$ & 10 & $230-232$ & 88 \\
$\mathbf{3 j}$ & $4-\mathrm{ClC}_{6} \mathrm{H}_{4}$ & $2,4-\mathrm{Cl}_{2} \mathrm{C}_{6} \mathrm{H}_{3}$ & 10 & $159-161$ & 85 \\
\hline
\end{tabular}

All the products were new, which were characterized by IR and ${ }^{1} \mathrm{H}$ NMR analysis. To identify the structure of the products further, we also provide a structural study for compound 3a by X-ray crystallography (Figure 1).

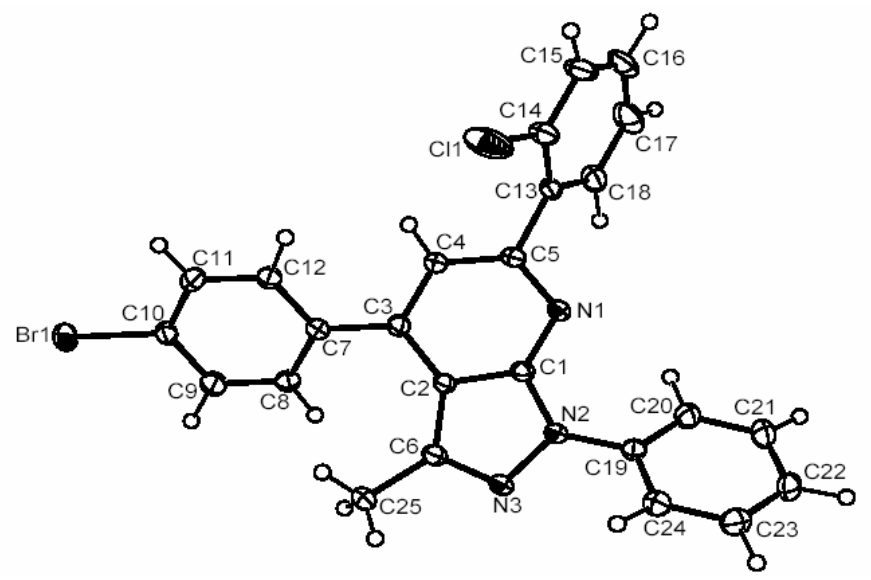

Figure 1. Molecular structure of 3a.

\section{Experimental Section}

General Procedures. Microwave irradiation was carried out in a modified commercial microwave oven (2450 MHz, Nanjing Sanle) under atmospheric pressure. Melting points were determined in open capillaries and are uncorrected. The IR spectra were recorded on a Shimadzu spectrometer. ${ }^{1} \mathrm{H}$ NMR spectra were measured on a DPX 400 spectrometer operating at 400 $\mathrm{MHz}$, using DMSO- $d_{6}$ as solvent and TMS as internal standard. Elemental analyses were determined by using a Perkin-Elmer 240c elemental analysis instrument. 


\section{General experimental procedure}

A dry flask $(25 \mathrm{~mL})$ was charged with aminopyrazole 1 (1 mmol), chalcone 2 (1 mmol), glycol $(2 \mathrm{~mL})$ and catalyst $\mathrm{ZnCl}_{2}(0.05 \mathrm{mmol})$. The flask was then connected with refluxing equipment. After microwave irradiation for 8-12 min, the reaction mixture was cooled and washed with ethanol. The crude products were purified by recrystallization from $95 \%$ ethanol to afford 3 .

4-(4-Bromophenyl)-6-(2-chlorophenyl)-3-methyl-1-phenyl-1H-pyrazolo[3,4-b]pyridine (3a). IR (KBr, v, cm $\left.{ }^{-1}\right): 3061,2963,2924,1677,1595,1574,1505,1429,1346,1289,1146,1062$, 1212, 827, 761, 695, 675, 639. ${ }^{1} \mathrm{H}$ NMR (400 MHz, DMSO- $\left.d_{6}\right): \delta 2.33\left(3 \mathrm{H}, \mathrm{s}, \mathrm{CH}_{3}\right), 7.33 \sim 8.31$ $(13 \mathrm{H}, \mathrm{m}, \mathrm{Ar}-\mathrm{H}), 7.48(1 \mathrm{H}, \mathrm{s}, \mathrm{C}-\mathrm{H})$. Anal. Calcd for $\mathrm{C}_{25} \mathrm{H}_{17} \mathrm{BrClN}_{3}$ : C, 63.24; H, 3.61; N, 8.85. Found: C, 63.08; H, 3.63; N, 8.78.

4-(4-Bromophenyl)-3-methyl-1-phenyl-6-p-tolyl-1H-pyrazolo[3,4-b]pyridine (3b). IR ( $\mathrm{KBr}$, $\left.\mathrm{v}, \mathrm{cm}^{-1}\right)$ : 3060, 3027, 2911, 2857, 1595, 1574, 1504, 1436, 1417, 1338, 1309, 1148, 1057, 1011, 854, 819, 762, 677, 634. ${ }^{1} \mathrm{H}$ NMR (400 MHz, DMSO- $\left.d_{6}\right): \delta 2.40\left(3 \mathrm{H}, \mathrm{s}, \mathrm{CH}_{3}\right), 2.29\left(3 \mathrm{H}, \mathrm{s}, \mathrm{CH}_{3}\right)$, 7.33-8.39 (14H, m, Ar-H, and C-H). Anal. Calcd for $\mathrm{C}_{26} \mathrm{H}_{20} \mathrm{BrN}_{3}: \mathrm{C}, 68.73 ; \mathrm{H}, 4.44 ; \mathrm{N}, 9.25$. Found: C, 68.61; H, 4.41; N, 9.28.

4-(4-Fluorophenyl)-3-methyl-1-phenyl-6-p-tolyl-1H-pyrazolo[3,4-b]pyridine (3c). IR ( $\mathrm{KBr}$, $\left.\mathrm{v}, \mathrm{cm}^{-1}\right): 3063,3034,2919,2854,1596,1565,1510,1411,1347,1223,1155,1047,1011,841$, 820, 756, 692, 641. ${ }^{1} \mathrm{H}$ NMR (400 MHz, DMSO-d 6$): \delta 2.40\left(3 \mathrm{H}, \mathrm{s}, \mathrm{CH}_{3}\right), 2.28\left(3 \mathrm{H}, \mathrm{s}, \mathrm{CH}_{3}\right)$, 7.37-8.39 (14H, m, Ar-H, and C-H). Anal. Calcd for $\mathrm{C}_{26} \mathrm{H}_{20} \mathrm{FN}_{3}$ : C, 79.37; H, 5.12; N, 10.68. Found: C, 79.21; H, 5.11; N, 10.62.

\section{4-(3,4-Dichlorophenyl)-6-(4-methoxyphenyl)-3-methyl-1-phenyl-1H-pyrazolo[3,4-}

b]pyridine (3d). IR ( $\left.\mathrm{KBr}, \mathrm{v}, \mathrm{cm}^{-1}\right)$ : 3059, 2987, 2932, 2835, 1670, 1597, 1570, 1504, 1468, $1347,1308,1247,1235,1174,1027,830,755,695,637 .{ }^{1} \mathrm{H}$ NMR (400 MHz, DMSO- $\left.d_{6}\right): \delta 2.28$ $\left(3 \mathrm{H}, \mathrm{s}, \mathrm{CH}_{3}\right), 3.86\left(3 \mathrm{H}, \mathrm{s}, \mathrm{OCH}_{3}\right), 7.59-8.38(12 \mathrm{H}, \mathrm{m}, \mathrm{Ar}-\mathrm{H}), 7.81(1 \mathrm{H}, \mathrm{s}, \mathrm{C}-\mathrm{H})$. Anal. Calcd for $\mathrm{C}_{26} \mathrm{H}_{19} \mathrm{Cl}_{2} \mathrm{~N}_{3} \mathrm{O}: \mathrm{C}, 67.83 ; \mathrm{H}, 4.16 ; \mathrm{N}, 9.13$. Found: $\mathrm{C}, 67.76 ; \mathrm{H}, 4.13 ; \mathrm{N}, 9.18$.

6-(4-Methoxyphenyl)-3-methyl-4-(3-nitrophenyl)-1-phenyl-1H-pyrazolo[3,4-b]pyridine (3e). IR $\left(\mathrm{KBr}, \mathrm{v}, \mathrm{cm}^{-1}\right): 3059,2996,2925,2835,1674,1594,1573,1526,1505,1415,1347,1299$, 1182, 1150, 1031, 839, 758, 729, 691, 638. ${ }^{1} \mathrm{H}$ NMR (400 MHz, DMSO- $\left.d_{6}\right): \delta 2.26\left(3 \mathrm{H}, \mathrm{s}, \mathrm{CH}_{3}\right)$, $3.86\left(3 \mathrm{H}, \mathrm{s}, \mathrm{OCH}_{3}\right), 7.11-8.54(13 \mathrm{H}, \mathrm{m}, \mathrm{Ar}-\mathrm{H}), 7.90(1 \mathrm{H}, \mathrm{s}, \mathrm{C}-\mathrm{H})$. Anal. Calcd for $\mathrm{C}_{26} \mathrm{H}_{20} \mathrm{~N}_{4} \mathrm{O}_{3}$ : C, 71.55; H, 4.62; N, 12.84. Found: C, 71.47; H, 4.63; N, 12.79.

4-(4-Chlorophenyl)-6-(2-methoxyphenyl)-3-methyl-1-phenyl-1H-pyrazolo[3,4-b]pyridine (3f). IR (KBr, v, cm $\left.{ }^{-1}\right): 3048,3007,2955,2929,2833,1597,1572,1505,1490,1415,1345$, 1242, 1173, 1147, 1080, 1031, 1014, 825, 755, 688, 642. ${ }^{1} \mathrm{H}$ NMR (400 MHz, DMSO-d 6 ): $\delta 2.26$ $\left(3 \mathrm{H}, \mathrm{s}, \mathrm{CH}_{3}\right), 3.86\left(3 \mathrm{H}, \mathrm{s}, \mathrm{OCH}_{3}\right), 7.10-8.39(14 \mathrm{H}, \mathrm{m}, \mathrm{Ar}-\mathrm{H}$ and $\mathrm{C}-\mathrm{H})$. Anal. Calcd for $\mathrm{C}_{26} \mathrm{H}_{20} \mathrm{ClN}_{3} \mathrm{O}: \mathrm{C}, 73.32 ; \mathrm{H}, 4.73 ; \mathrm{N}, 9.87$. Found: $\mathrm{C}, 73.39 ; \mathrm{H}, 4.70 ; \mathrm{N}, 9.82$.

4-(4-Methoxyphenyl)-3-methyl-1-phenyl-6-p-tolyl-1H-pyrazolo[3,4-b]pyridine (3g). IR $\left(\mathrm{KBr}, \mathrm{v}, \mathrm{cm}^{-1}\right)$ : 3033, 2998, 2958, 2917, 2833, 1592, 1575, 1514, 1415, 1348, 1284, 1243, 1177 , $1145,1031,970,906,819,752,700,642,610 .{ }^{1} \mathrm{H}$ NMR (400 MHz, DMSO- $\left.d_{6}\right): \delta 2.31(3 \mathrm{H}, \mathrm{s}$, $\left.\mathrm{CH}_{3}\right), 2.40\left(3 \mathrm{H}, \mathrm{s}, \mathrm{CH}_{3}\right), 3.88\left(3 \mathrm{H}, \mathrm{s}, \mathrm{OCH}_{3}\right), 7.14-8.40(13 \mathrm{H}, \mathrm{m}, \mathrm{Ar}-\mathrm{H}), 7.72(1 \mathrm{H}, \mathrm{s}, \mathrm{C}-\mathrm{H})$. Anal. Calcd for $\mathrm{C}_{27} \mathrm{H}_{23} \mathrm{~N}_{3} \mathrm{O}: \mathrm{C}, 79.97 ; \mathrm{H}, 5.72 ; \mathrm{N}, 10.36$. Found: C, 79.88; H, 5.70; N, 10.32 . 
4-(4-Chlorophenyl)-6-(4-fluorophenyl)-3-methyl-1-phenyl-1H-pyrazolo[3,4-b]pyridine (3h). IR $\left(\mathrm{KBr}, v, \mathrm{~cm}^{-1}\right)$ : 3050, 2993, 2964, 2913, 1677, 1596, 1575, 1504, 1349, 1226, 1160, 1089 , 1050, 1014, 826, 752, 689, 638. ${ }^{1} \mathrm{H}$ NMR (400 MHz, DMSO-d $): \delta 2.28\left(3 \mathrm{H}, \mathrm{s}, \mathrm{CH}_{3}\right), 7.34-8.37$ (13H, m, Ar-H), $7.81(1 \mathrm{H}, \mathrm{s}, \mathrm{C}-\mathrm{H})$. Anal. Calcd for $\mathrm{C}_{25} \mathrm{H}_{17} \mathrm{ClFN}_{3}$ : C, 72.55; H, 4.14; N, 10.15. Found: C, 72.46; H, 4.15; N, 10.11 .

6-(2-Chlorophenyl)-4-(4-methoxyphenyl)-3-methyl-1-phenyl-1H-pyrazolo[3,4-b]pyridine (3i). IR (KBr, v, cm $\left.{ }^{-1}\right): 3049,3006,2957,2929,2839,1681,1657,1608,1596,1571,1501$, 1462, 1434, 1343, 1033, 1245, 1175, 1151, 1032, 837, 758, 688, 642. ${ }^{1} \mathrm{H}$ NMR (400 MHz, DMSO-d $\left.)_{6}\right): \delta 2.36\left(3 \mathrm{H}, \mathrm{s}, \mathrm{CH}_{3}\right), 3.87\left(3 \mathrm{H}, \mathrm{s}, \mathrm{OCH}_{3}\right), 7.14-8.33(13 \mathrm{H}, \mathrm{m}, \mathrm{Ar}-\mathrm{H}), 7.42(1 \mathrm{H}, \mathrm{s}, \mathrm{C}-$ H). Anal. Calcd for $\mathrm{C}_{26} \mathrm{H}_{20} \mathrm{ClN}_{3} \mathrm{O}: \mathrm{C}, 73.32 ; \mathrm{H}, 4.73 ; \mathrm{N}, 9.87$. Found: C, 73.25; H, 4.72; N, 9.83. 4-(4-Chlorophenyl)-6-(2,4-dichlorophenyl)-3-methyl-1-phenyl-1H-pyrazolo[3,4-b]pyridine (3j). IR (KBr, v, $\mathrm{cm}^{-1}$ ): 3048, 2956, 2920, 1598, 1573, 1505, 1489, 1436, 1342, 1287, 1150, 1086, 1013, 855, 815, 758, 682, 630. ${ }^{1} \mathrm{H}$ NMR (400 MHz, DMSO- $\left.d_{6}\right): \delta 2.32\left(3 \mathrm{H}, \mathrm{s}, \mathrm{CH}_{3}\right), 7.22-$ $8.30(12 \mathrm{H}, \mathrm{m}, \mathrm{Ar}-\mathrm{H}), 7.49$ (1H, s, C-H). Anal. Calcd for $\mathrm{C}_{25} \mathrm{H}_{16} \mathrm{Cl}_{3} \mathrm{~N}_{3}$ : C, 64.61; H, 3.47; N, 9.04. Found: C, 64.53; H, 3.46; N, 9.07.

X-ray structure determination of 3a. Colourless prisms, $\mathrm{C}_{25} \mathrm{H}_{17} \mathrm{BrClN}_{3}, M r=474.78$, Triclinic, space group $P-1, a=9.3798(11), b=10.6200(13), c=11.7433(15) \AA, \alpha=72.932(9), \beta=78.877(10)$, $\gamma=72.045(9)^{\circ}, \quad V=1057.0(2) \mathrm{A}^{3}, \quad Z=2, \quad D_{c}=1.492 / \mathrm{cm}^{3}, \quad \mu=2.088 \mathrm{~mm}^{-1}, \quad F(000)=480$, crystal dimensions $0.60 \times 0.30 \times 0.25 \mathrm{~mm}^{3}$. Intensity data were collected using a Rigaku Mercury diffractometer at $193 \mathrm{~K}$, graphite monochromator Mo K $\alpha$ radiation $(\lambda=0.7107 \AA)$, using the $\omega-2 \theta$ scan technique to a maximum $2 \theta$ of $54.96^{\circ}$. A total of 11838 reflections were collected with 4743 unique ones $\left(R_{\text {int }}=0.0278\right)$, of which 4275 reflections were observed with $I>2 \sigma(I)$. The final $R$ and $w R$ values were 0.0428 and $0.0879, s=1.062,(\Delta / \sigma)_{\max }=0.001$. The maximum peak and minimum peak in the final difference map is 0.813 and $-0.759 \mathrm{e} / \AA^{3}$.

\section{Acknowledgements}

We thank the National Natural Science Foundation of China (No. 20372057), and the Nature Science Foundation of the Jiangsu Province (No. BK2001142) and the Nature Science Foundation of Jiangsu Education Department (No. 01KJB150008) and the Key lab. of Biotechnology for Medicinal Plants of Jiangsu Province for financial supports.

\section{References}

1. Sekikawa, I.; Nishie, J.; Tono-oka, S.; Tanaka, Y.; Kakimoto, S.; J. Heterocycl. Chem. 1973, 10,931.

2. Kukzynski, L.; Mrizikiewic, A.; Banaszkiewicz, W.; Pol, K. P. J. Pharmacol. Pharm. 1979, $31,217$. 
3. Kamal, A. M.; Atalla, A. A.; Mohamed, T. A.; Geies, A. A.; Naturforsch. Z. B: Chem. Sci. 1991, 46, 541.

4. Chen, Y. L. International Patent WO 9534563 AL 1995; Chem. Abstr. 1995, 124, 232447.

5. Orlov, V. D.; Quiroga, J.; Kolos, N. N. Khim. Geterosikl. Soedin 1987, 1247.

6. Orlov, V. D.; Quiroga, J.; Kolos, N. N.; Desenko, S. M. Khim. Geterosikl. Soedin 1988, 962.

7. Quiroga, J.; Insuasty, B.; Rincón, R.; Larrahondo, M.; Hanold, N.; Meier, H. J. Heterocycl. Chem. 1994, 31, 1333.

8. Quiroga, J.; Insuasty, B.; Marín, M.; Aguirre, A.; Meier, H. Rev. Col. Quim. 1992, 21, 29.

9. Quiroga, J.; Hormaza, A.; Insuasty, B.; Saitz, C.; Jullian, C.; Cańete, A. J. Heterocycl. Chem. 1998, 35, 61.

10. Quiroga, J.; Insuasty, B.; Cruz. S.; Hernandez, P.; Bolańos, A.; Moreno, R.; Hormaza, A.; Almeida, R. H. J. Heterocycl. Chem. 1998, 35, 333.

11. Quiroga, J.; Hormaza, A.; Insuasty, B.; Marquez, M.; J. Heterocycl. Chem. 1998, 35, 409.

12. Quiroga, J.; Hormaza, A.; Insuasty, B.; Saitz, C.; Jullian, C. J. Heterocycl. Chem. 1998, 35, 575.

13. Quiroga, J.; Insuasty, B.; Hormaza, A.; Gamenara, D.; Domínguez, L.; Saldańa, J. J. Heterocycl. Chem. 1999, 36, 11.

14. Quiroga, J.; Insuasty, B.; Hormaza, A.; Cabildo, P.; Claramunt, R. M.; Elguero, J. Heterocycl. Commun. 1999, 5, 115.

15. Feng S.; Shujiang T.; Fang F.; Tuanjie L. Arkivoc 2005, (i), 137. 